\title{
LINFOMA DIFUSO DE CÉLULAS B GRANDES: ¿UNA SOLA ENFERMEDAD?
}

\author{
Paulo Castañeda-Ruiz ${ }^{1 a}$, Fanny Via y Rada ${ }^{1 a}$, Rebeca Serra-Jaramillo ${ }^{3 a}$, Eduardo Paz-Cornejo ${ }^{2 a}$, \\ Fernando Salas-Sánchez ${ }^{3 a}$
}

\begin{abstract}
RESUMEN
Los linfomas no Hodgkin, trastornos hematológicos de alta incidencia, constituyen a su vez, un grupo heterogéneo de neoplasias, dentro de las cuales los linfomas difusos de células B grandes (LDCBG) representan un porcentaje superior al $60 \%$ en la mayoría de las series nacionales e internacionales. Estos LDCBG, a su vez, se presentan con características clínicas, perfiles genéticos y respuestas terapéuticas variadas, apreciándose específicamente en relación a su respuesta, que en un subgrupo de ellos se obtienen tasas de sobrevidas libre de progresión, sobrevidas totales y respuestas objetivas adecuadas, y en otro subgrupo se obtienen respuestas de calidad inferior. Por lo que es necesario el diseño de esquemas de tratamiento basados en criterios clínicos, inmunohistoquímicos y de perfil genético, que los agrupen adecuadamente para identificar modalidades terapéuticas diferenciadas para cada uno de ellos y así mejorar e igualar las tasas de respuesta mencionadas. En la actualidad, estas neoplasias son catalogadas como linfomas de alto y bajo riesgo y se están desarrollando esquemas terapéuticos diferentes al R-CHOP convencional, como el R-DA-EPOCH, R2CHOP, IR-CHOP, VR-CHOP y el eveRCHOP que se espera que presenten resultados alentadores.
\end{abstract}

Palabras clave: Linfoma difuso de células B grandes; Terapia biológica; Sobrevida (fuente: DeCS BIREME).

\section{DIFFUSE LARGE B-CELL LYMPHOMA: A SINGLE DISEASE?}

\begin{abstract}
Non-Hodgkin's lymphomas are common and comprise a heterogeneous group of neoplasms, with diffuse large B-cell lymphoma (DLBCL) accounting for more than $60 \%$ of cases. Moreover, DLBCL presents with a variety of clinical characteristics, genetic profiles, and therapeutic responses. We aimed to improve progression-free survival, total survival, and objective results in an DLBCL subgroup. However, another subgroup demonstrated poor responses. Therefore, it is necessary to design treatment programs based on clinical, immunohistochemical, and genetic profiles, grouping cases properly to identify correct therapeutic modalities for each lymphoma subtype. Currently, these neoplasms are classified as high- and low-risk lymphomas, and therapeutic regimens are being developed to complement conventional R-CHOP, such as R-DA-EPOCH, R2CHOP, IR-CHOP, VR-CHOP, and eveRCHOP, which are expected to improve outcomes.
\end{abstract}

Key words: Diffuse large B cell lymphoma; Biology therapy; Survival (source: MeSH NLM).

\section{INTRODUCCIÓN}

El linfoma no Hodgkin (LNH), neoplasia maligna frecuente a nivel mundial, es un conjunto de entidades que tienen una alta incidencia y prevalencia entre las diversas neoplasias. De hecho, la Agencia Internacional para Investigación en Cáncer (IARC) en su proyecto GLOBOCAN 2012 la ubica dentro de las diez neoplasias más frecuentes en todo el mundo, con un rango que varía entre las diversas regiones geográficas (séptimo lugar para América del
Norte y octavo para América del Sur) ${ }^{(1)}$. Dentro de esta patología, el linfoma no Hodgkin difuso de células B grandes (LDCBG) es la neoplasia linfoide más frecuente en adultos, correspondiendo a un $30-40 \%$ de todos los $\mathrm{LNH}$ a nivel mundial ${ }^{(2-3)}$.

En cuanto a la situación epidemiológica de los linfomas en el Perú, esta neoplasia maligna en conjunto ocupa el cuarto lugar en frecuencia de cáncer, por debajo de las neoplasias de cérvix, mama y estómago, con una incidencia anual de

\footnotetext{
Hospital Nacional Guillermo Almenara Irigoyen. Lima, Peru

Hospital Nacional Alberto Sabogal Sologuren. Lima, Peru

Clinica Oncosalud. Lima, Peru

Médico especialista en oncología medica

Recibido: 05/04/2017 Aprobado: 06/09/2017 En línea: 29/09/2017
}

Citar como: Castañeda-Ruiz P, Via y Rada F, Serra-Jaramillo R, Paz-Cornejo E, Salas-Sánchez F. Linfoma difuso de células B grandes: ¿una sola enfermedad? Rev Peru Med Exp Salud Publica. 2017;34(3):551-9. doi: 10.17843/rpmesp.2017.343.2803 
$\mathrm{LNH}$, que ha ido incrementándose en los últimos años, según los datos reportados por el Instituto Nacional de Enfermedades Neoplásicas (INEN) (4) (Figura 1A). En nuestro país existe una proporción similar entre hombres y mujeres, y un rango de edad de presentación muy amplio, con un promedio de 50 años. Además, específicamente el LDCBG, representa aproximadamente un $40 \%$ de los LNH y un $60-65 \%$ de los $\mathrm{LNH}$ de células $\mathrm{B}$, según los datos epidemiológicos de los hospitales Cayetano Heredia y Arzobispo Loayza ${ }^{(5-6)}$.

EI LDCBG se clasifica en dos grupos: los no especificados (NOS), que representa la gran mayoría de casos, y los especificados, dentro de los cuales se tienen los siguientes subtipos: rico en histiocitos/células T, primario del SNC, primario cutáneo tipo pierna, positivo al Epstein Barr virus NOS y asociado con inflamación crónica ${ }^{(7)}$; cada uno de estos últimos con sus propias y especificas características clínicopatológicas.

EI LDCBG NOS es, a su vez, un grupo muy heterogéneo con respecto a diversas características como la morfología celular, presentación clínica, biología y respuesta al tratamiento, características todas que se ven reflejadas en el amplio espectro de respuestas obtenidas con la terapia estándar ${ }^{(8)}$. Hasta hace algunos años, el tratamiento único, estándar y adecuado era un esquema de inmunoquimioterapia basado en antraciclinas (R-CHOP). Este régimen consigue una tasa de curación del $60-70 \%$ a nivel mundial; apreciándose, sin embargo, que a pesar de estar al frente de una patología potencialmente curable, un porcentaje importante de pacientes $(30-40 \%)$ serán refractarios al tratamiento o recurrirán en los primeros dos años de iniciado el mismo ${ }^{(2,3,8)}$. Con el avance de la tecnología molecular y las nuevas luces que nos han traído los perfiles de expresión genética, se han desarrollado nuevas estrategias para estratificar y subclasificar a esta enfermedad en grupos más homogéneos que permitan desarrollar enfoques terapéuticos diferenciados y así superar las tasas de curación actuales, aproximándonos a un control de la enfermedad cercano al $100 \%$.

Esta diversidad de conformaciones biomoleculares, con heterogeneidad clínica y de respuesta terapéutica, hace importante una revisión de los LDCBG, principalmente del grupo NOS, el cual nos dedicaremos a desarrollar en el presente artículo, para un correcto entendimiento del comportamiento de esta neoplasia y de la necesidad del empleo de estrategias diferenciadas para su manejo.

\section{ESTRATEGIA DE BÚSQUEDA Y CRITERIOS DE SELECCIÓN DE ARTÍCULOS}

Para elaborar el presente artículo se revisó la literatura disponible en Medline, a través del buscador Pubmed (https://www.ncbi.nlm.nih.gov/pubmed/). Se usó términos de búsqueda en español e inglés. Se incluyeron estudios originales y revisiones que hayan evaluado o discutido la clasificación y/o tratamiento de los LDCBG hasta diciembre de 2016. Se excluyeron aquellos artículos que brindaban opiniones, conclusiones, o recomendaciones no sustentadas por estudios de investigación.

\section{CLASIFICACIÓN DEL LDCBG ADAPTADO AL RIESGO}

Existen varias formas de subclasificar al LDCBG desde el punto de vista teórico (clasificaciones morfológicas, moleculares e inmunohistoquímicas, entre otras) ${ }^{(7)}$. Sin embargo, la necesidad de mejorar las tasas de respuesta y curación actuales, requiere formular una subclasificación práctica que se correlacione con el pronóstico y permita agendar estrategias terapéuticas diferenciadas con el fin de vencer la heterogeneidad en la respuesta ${ }^{(8)}$.

Una de las más recientes clasificaciones del LDCBG es de acuerdo a la célula de origen de estas neoplasias. En esta, los LDCBG pueden ser agrupados como neoplasias derivadas de los centros germinales (GCB), o de células $B$ activadas $(A B C)$ y, en un tercer subtipo no clasificable ${ }^{(3,9)}$. Esta clasificación ha demostrado mediante varios estudios, delimitar subgrupos de linfomas con pronóstico y respuesta diferente al tratamiento estándar, siendo el $\mathrm{ABC}$ el subgrupo de comportamiento más agresivo $\mathrm{y}$, por ende, de peor pronóstico ${ }^{(2,9,10)}$ (Figura 1B).

Para la determinación de la célula de origen, las técnicas que precisan el perfil de expresión genética (GEP) constituye el método clásicoy más exactoen laactualidad ${ }^{(3,111}$. Sinembargo, debido a su poca practicidad y alto costo, fue necesaria la búsqueda de alternativas que permitieran una aplicación cotidiana con una alta concordancia tanto para predecir el comportamiento clínico como las tasas de respuesta. Es así que, con el empleo de la inmunohistoquímica se han desarrollado los algoritmos de Hans y Choi (los cuales usan 3 y 5 marcadores respectivamente), que son comúnmente utilizados y que han logrado una alta concordancia con el GEP $(86 \% \text { y } 87-93 \% \text { respectivamente) })^{(3,9,10)}$, facilitando asi su uso rutinario y a costos asequibles (Figura 2).

Al respecto, debemos precisar que, a diferencia del algoritmo de Choi, el de Hans, si bien no permite la discriminación exacta entre GCB y ABC, divide los LDCBG en dos grupos: GCB y no GCB (esta última agrupando los $A B C$ y los no clasificables) que en la práctica tienen una utilidad muy similar a la clasificación original. Por ello, debido a su simplicidad, por el menor número de marcadores empleados y por su implementación técnica estandarizada, el algoritmo de Hans es el más aceptado y usado a nivel internacional ${ }^{(7,10)}$. 
A

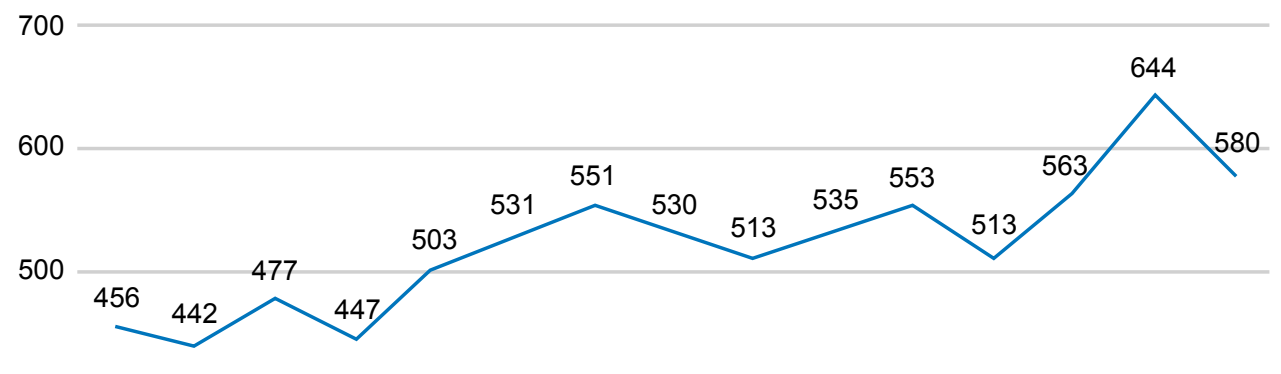

400

300

200020012002200320042005200620072008200920102011201220132014

B
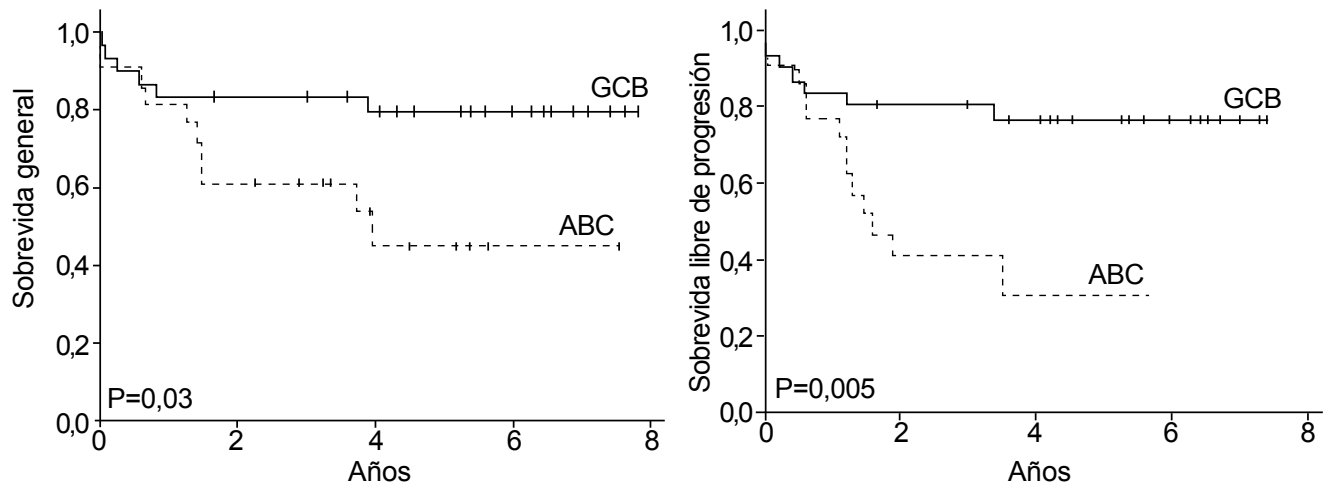

A) Número de casos incidentes de linfomas no Hodgkin en el Instituto Nacional de Enfermedades Neoplásicas (INEN) del 20002014. (Fuente: Datos epidemiológicos INEN). B) Sobrevida general y sobrevida libre de progresión del Linfoma difuso de células B grandes tratado con inmunoquimioterapia dividido de acuerdo al subtipo molecular determinado por perfil de expresión genética. $\mathrm{GCB}=$ células $\mathrm{B}$ de centro germinal. $\mathrm{ABC}=$ células $\mathrm{B}$ activadas

Figura 1. Estado epidemiológico y pronóstico actual del linfoma no Hodgkin

Adicional al criterio genético-molecular, existe el índice pronóstico internacional (IPI), que permite, de acuerdo a un score predeterminado, subdividir a los linfomas en neoplasias de riesgo bajo, intermedio-bajo, intermedio-alto $\mathrm{y}$ alto ${ }^{(12)}$. Igualmente, un marcador que refleja el índice de proliferación celular como el Ki67, ha sido empleado también en varios estudios para agrupar a los linfomas, en dos categorías: la de alto riesgo con un Ki67 alto: $\geq 70$ $80 \%$ (dependiendo la literatura) y la de riesgo bajo con el Ki67 bajo: $<70-80 \%$ y han demostrado su importancia en el pronóstico de este tipo de neoplasias ${ }^{(13-14)}$.

Es importante mencionar que el desarrollo y la comprensión de las vías moleculares relacionadas a la proliferación celular y apoptosis, como son las que comprenden los protooncogenes MYC y BCL2, y del gen supresor tumoral BCL6, ha permitido la identificación de dos subgrupos particulares que se conocen como los linfomas doble/triple hit y los doble expresores. Los linfomas doble hit son un subgrupo de LDCBG NOS que tienen rearreglos en los genes MYC y BCL2 o BCL6 que los hacen altamente proliferativos y agresivos con una mala respuesta al tratamiento estándar y pronóstico desfavorable. En su mayoría corresponden a un LDCBG centro germinal (GCB) y la asociación más común es la mutación del gen MYC y BCL2. Si el LDCBG presenta mutaciones en los tres genes al mismo tiempo (MYC, BCL2 y BCL6) se denomina triple hit ${ }^{(15,16)}$. Los otros, los llamados linfomas doble expresores, son LDCBG que sobreexpresan las proteínas MYC y BCL2, los cuales no siempre se corresponden con un rearreglo de los genes homónimos, correspondiendo en su mayoría a los LDCBG $A B C$, con una respuesta y pronóstico peor que los GCB que no sobreexpresan estas proteínas, pero mejor que los doble/ triple hit (Figura 3). Es necesario precisar que, generalmente, para definir sobreexpresión se necesita una tinción igual o mayor al $40 \%$ para MYC y $70 \%$ para BCL2 ${ }^{(17)}$. 


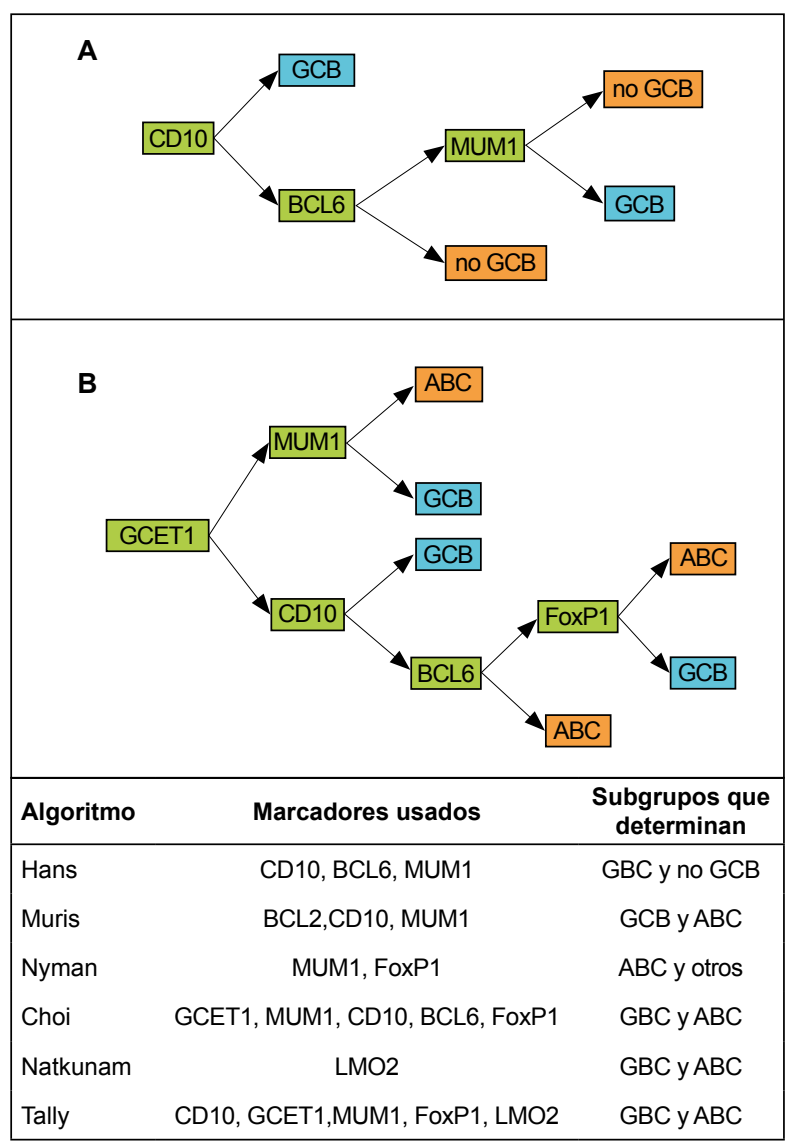

A) Algoritmo de Hans, basado en 3 marcadores

B) B) Algoritmo de Choi, basado en 5 marcadores

$\mathrm{GCB}=$ células $\mathrm{B}$ de centro germinal. $\mathrm{ABC}=$ células $\mathrm{B}$ activadas

Figura 2. Principales algoritmos inmunohistoquímicos para la clasificación molecular del Linfoma difuso de células B grandes ${ }^{(18)}$

Guiándonos en todos estos conceptos precedentes, y en un intento de unificar criterios usando los conocimientos actuales, consideramos que la mejor propuesta para clasificar hoy en día a los LDCBG NOS es la de agruparlos en dos categorías: alto y bajo riesgo, clasificación que es la tendencia en la actualidad en los centros altamente especializados a nivel internacional, sobre todo americanos (Tabla 1) ${ }^{(18-20)}$.

Estos diferentes parámetros, la célula de origen, el IPI y el Ki67, que han demostrado un valor pronóstico claro y que han sido validados en diversos estudios ${ }^{(9,12-14)}$, permiten diferenciar los LDCBG en linfomas de bajo o alto riesgo, estos últimos con un pronóstico significativamente peor y que, por lo tanto, necesitarían un esquema terapéutico diferente para mejorar las tasas de respuesta obtenidas con los tratamientos convencionales como el R-CHOP, el cual es un esquema de inmunoquimioterapia basado en rituximab, doxorrubicina, ciclofosfamida, vincristina y prednisona, que es el tratamiento estándar de primera línea del LDCBG sin discriminación alguna, con excepción de los doble/triple hit en los cuales se estipula un tratamiento mas agresivo ${ }^{(21)}$.

En la literatura especializada encontramos nuevos esquemas en estudio que buscan lograr este objetivo, principalmente en los grupos con caracteristicas de alto riesgo, con evidencia científica cada vez mayor, lo cual nos brinda la posibilidad de que en un futuro no muy lejano se reemplace al $\mathrm{R}-\mathrm{CHOP}$ como tratamiento de primera linea en este grupo importante de pacientes. Nosotros comentaremos las principales terapias que actualmente se encuentran en estudio.

\section{DOSIS AJUSTADA DE R-EPOCH (R-DA-EPOCH)}

Una combinación de inmunoquimioterapia adicional, R-DA-EPOCH (rituximab más etoposido, prednisona, vincristina, ciclofosfamida, doxorubicina), esquema diseñado por Wyndham Wilson en el National Cancer Institute, demostró inicialmente ser beneficioso en el subgrupo de pacientes con linfoma no hodgkin de células B primario mediastinal, al no ser necesaria la radioterapia cuando se usa este régimen, y en los últimos años demostró ser una alternativa de eficacia similar al R-CHOP-21 en los pacientes con LDCBG y hasta hace poco sin diferencias estadísticamente significativas. Sin embargo, diversos estudios, están sugiriendo el beneficio de R-DA-EPOCH en los subgrupos de pacientes con características de alto riesgo y permitiendo igualar las tasas de respuesta obtenidas con el R-CHOP-21 en los pacientes de bajo riesgo ${ }^{(22,23)}$.

Desde el 2010, el Centro de Cáncer MD Anderson (https:// www.mdanderson.org/), ha venido usando el R-DAEPOCH para el tratamiento de pacientes con alto riesgo, y en la 58. ${ }^{a}$ reunión anual y exposición de la Sociedad Americana de Hematología (ASH 2016) presentaron una revisión retrospectiva de su uso durante 4 años para

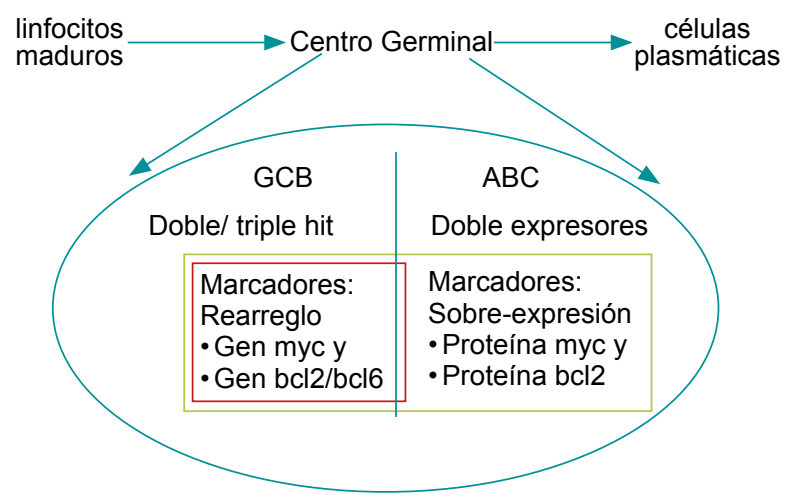

$\mathrm{GCB}=$ células $B$ de centro germinal. $A B C=$ células $B$ activadas.

Figura 3. Célula de origen y su relación con MYC y BCL2 en los Linfomas 
Tabla 1. Clasificación práctica de los linfomas difusos de células B grandes

\begin{tabular}{l}
\hline Linfoma de bajo riesgo \\
\hline Si presenta todas las siguientes características: \\
- De acuerdo a su célula de origen: $\mathrm{GCB}^{*}$ \\
- $\mathrm{IP}^{+}: 0-2$ \\
- ki67< $80 \%$ \\
- Proteínas $\mathrm{Bcl} 2$ y myc no sobre- expresadas ${ }^{\ddagger}$ \\
\hline
\end{tabular}

\section{Linfoma de alto riesgo}

Si presenta cualquiera de las siguientes características:

- De acuerdo a su célula de origen: No GCB

- IPI: 3-5

- ki67 $\geq 80 \%$

- Doble/triple hit

- Doble expresor

* La clasificación de acuerdo a su célula de origen está basado en el Algoritmo de Hans, GCB= células $B$ de centro germinal ${ }^{(17)}$

+ IPI: índice pronóstico internacional

₹ Definición de sobreexpresión por inmunohistoquímica: BCL2 $\geq 70 \%$, myc $\geq 40 \% 42$

pacientes nuevos con LDCBG, catalogados como de alto riesgo de acuerdo a los criterios estipulados previamente en la Tabla 1, con excepción de los dobles expresores y doble/triple hit, demostrando un incremento en las tasas de curación y logrando igualar la tasa de respuesta objetiva (TRO) y sobrevida global (SG) a los 3 años obtenidas en los pacientes de bajo riesgo tratados con R-CHOP-21(18), concluyendo que R-DA-EPOCH es un régimen altamente efectivo en pacientes con LDCBG de alto riesgo. Más aun, este centro oncológico, luego presentó un estudio comparativo de la respuesta al R-DA-EPOCH entre tres subgrupos de los linfomas de alto riesgo: doble/ triple hit, doble expresores y LDCBG de alto riesgo sin sobreexpresión o alteración de MYC y BCL2, encontrando una sobrevida libre de progresión (SLP) y SG al año, sin diferencia significativa entre los tres subgrupos (SG al año: $79 \%, 86 \%$ y $91 \%$ respectivamente, $p=0,3$. SLP al año: $72 \%, 65 \%$ y $87 \%$ respectivamente, $p=0,08)^{(19)}$.

Adicionalmente, en varios estudios retrospectivos publicados recientemente, se ha comparado el R-DA-EPOCH con esquemas más intensos, como R-HyperCVAD/MA y R-CODOX-M/IVAC en el manejo de pacientes con LDCBG doble hit con diversos resultados. Oki et al. (24), en un estudio retrospectivo de 10 años en el Centro de Cáncer MD Anderson publicado en el 2014, reportó una sobrevida libre de enfermedad (SLE) y SG a los 2 años superior para el R-DA-EPOCH en comparación con R-HyperCVAD/MA y R-CHOP (SLE: $67 \%$ vs $32 \%$ vs $25 \%$ respectivamente y SG: $76 \%$ vs $44 \%$ vs $41 \%$ respectivamente), con una respuesta completa (RC) de $68 \%$ para R-DA EPOCH y R-HyperCVAD/MA en comparación con el $40 \%$ con $\mathrm{R}-\mathrm{CHOP}$, coincidente con los resultados publicados en -el ASH 2016. Además, un metaanálisis publicado en el mismo año por Howlett et al. ${ }^{(25)}$ presentado en el ASH 2016, demostró que el R-DA- EPOCH estuvo asociado con una mejoría estadísticamente significativa en el SLP en comparación con el R-CHOP (27,8 meses vs 13,9 meses) y mejor SLP que los demás regímenes intensos $(27,8$ meses para R-DA-EPOCH vs 21,9 meses en promedio para los demás regímenes intensos), aunque no llega a ser significativo.

Actualmente existen estudios prospectivos en desarrollo que intentan tener una idea más clara del esquema óptimo en este subgrupo particular de linfomas de alto riesgo. Sin embargo, en base a la información obtenida hasta el momento, el tratamiento de elección ${ }^{(3)}$ y recomendado por la Sociedad Americana de Clínica Oncológica (ASCO, por sus siglas en inglés) (http://media4.asco.org/156/ edbook/2015_edbook.pdf) y en muchas escuelas a nivel internacional es R-DA-EPOCH, debido a que es mejor tolerado con un perfil de toxicidad menor que otros regímenes más intensos y con una eficacia similar o superior a estos últimos. Una posible explicación del beneficio del R-DA-EPOCH en los pacientes de alto riesgo, parece estar centrada en que las infusiones continuas de las drogas usadas en el manejo de estas neoplasias permiten vencer la resistencia de la célula linfática tumoral, lo que no ocurre cuando se realiza la administración de estas mismas drogas en bolo endovenoso en los esquemas clásicos como R-CHOP o R-CHOEP.

Un estudio Fase 3(CALGB-50303) se viene desarrollando actualmente para comparar y demostrar el beneficio del R-DA-EPOCH en este subgrupo de pacientes, en comparación con R-CHOP. Si bien es cierto, los resultados preliminares de este estudio presentados en la ASH 2016 no encontraron diferencias significativas entre ambos esquemas terapéuticos en relación a SG y SLP, esto ocurrió porque la comparación presentada fue considerando a todos los grupos de pacientes, sin agrupación alguna, quedando aún pendiente el análisis por grupos de riesgo, objetivo principal de este estudio prospectivo (26).

\section{ASOCIACIONES AL R-CHOP}

Los fármacos más estudiados en este tipo de linfomas son: lenalidomida, ibrutinib, bortezomib y everolimus ${ }^{(3)}$. Todas ellas con un mecanismo de acción relacionado a la vía del receptor de la célula $B(B C R)$, vía de señalización implicada en el desarrollo de diversos linfomas no Hodgkin (Figura 4A).

\section{R2CHOP}

La lenalidomida $(R)$, una droga inmunomoduladora derivada de la talidomida, ha demostrado eficacia 
importante en estudios fase 1 y 2 cuando se agrega al $\mathrm{R}-\mathrm{CHOP}$ para pacientes de alto riesgo, pero sin considerar el IPI y el Ki67 y con perfiles de toxicidad aceptables ${ }^{(27-29)}$. En el 2014, en el estudio REAL07 ${ }^{(27)}$ uno de los primeros trabajos multicéntricos fase 2 que introdujo la lenalidomida asociada al R-CHOP para mejorar las tasas de respuesta de los pacientes en alto riesgo, se intentó demostrar la eficacia y seguridad de esta combinación. El estudio encontró una RC de $81 \%$ para GCB y $89 \%$ para no GCB. El SLG y SG a los dos años fue de $71 \%$ vs $81 \%(p=0,7)$ y $94 \%$ vs $88 \%(p=0,58)$, respectivamente, sugiriendo, por tanto, una mejor eficacia para los no GCB. Un año más tarde, Nowakowski ${ }^{(28)}$ en un estudio de fase 2 , demostró también el beneficio de la adición de lenalidomida al R-CHOP en pacientes con LDCBG no GCB, elevando las pobres tasas de respuesta e igualando el SLP y SG a los 24 meses obtenidos en el grupo GCB $60 \%$ vs $59 \%(p=0,083)$ y $83 \%$ vs $75 \%$, $(p=0,61)$ respectivamente, concluyendo que el R2CHOP muestra una eficacia prometedora para mitigar el impacto negativo de los resultados de los pacientes con subtipo no GCB (Figura 4B). Actualmente, se viene desarrollando un estudio Fase 3 (ROBUST), que intenta finalmente evaluar la respuesta y utilidad de la lenalidomida en pacientes con LDCBG ABC determinado por GEP y cuyos resultados que saldrán en los próximos años, consolidarían a esta asociación

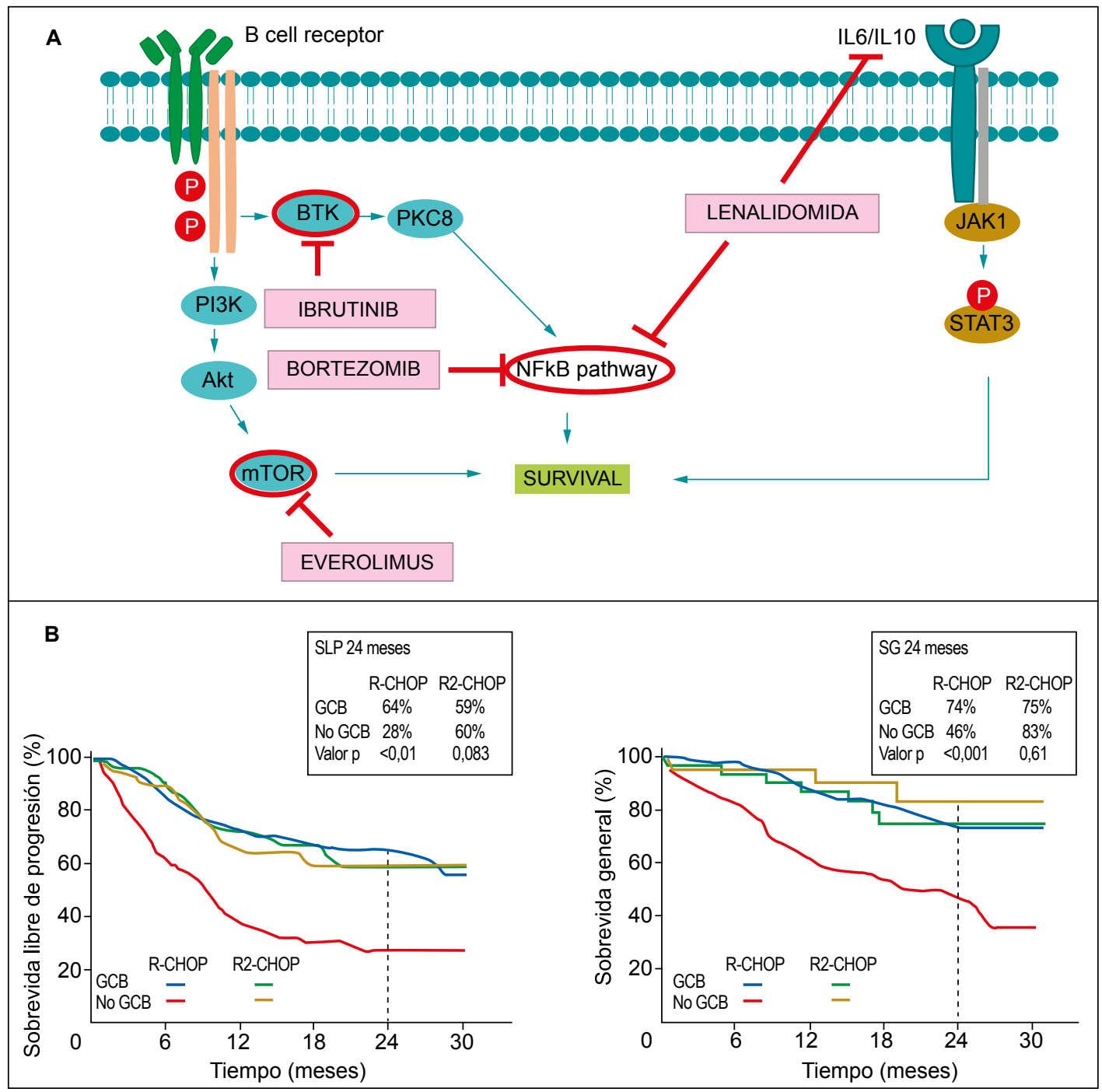

Adaptación con los resultados del estudio Fase II de Nowakowski et al. ${ }^{(28)}$. Izquierda: sobrevida libre de progresión (SLG) en pacientes tratados con R-CHOP y R2CHOP en LDCBG GCB vs no GCB. Derecha: sobrevida general (SG) en pacientes tratados con R-CHOP y R2CHOP en LDCBG GCB vs no GCB

LDCBG: linfoma difuso de células $B$ grandes difuso, CGB: células $B$ de centro germinal

Figura 4. A) Niveles de acción de los principales fármacos en desarrollo, B) Resultados del estudio comparativo entre R-CHOP y R2CHOP para pacientes nuevos con LDCGB GCB vs no GCB 
como una alternativa eficiente en primera línea en este subgrupo no GCB de linfomas de alto riesgo ${ }^{(30)}$.

\section{IR-CHOP}

El ibrutinib es un inhibidor de BKT (Bruton's tyrosine kinase), proteína que cumple una función importante en la vía de señalización del BCR para la maduración de la célula $B$. En un estudio fase $1 b$ se investigó la seguridad y eficacia de la combinación de Ibrutinib con R-CHOP (IR-CHOP) en pacientes con LNH de células B nunca tratados ${ }^{(31)}$, demostrando un perfil de toxicidad aceptable y una buena tolerancia de la combinación (efecto adverso serio más frecuente fue neutropenia febril en un $18 \%$ de los pacientes). Este fármaco ha sido investigado también en estudios fase $2^{\left({ }^{(32)}\right.}$ en LDCBG recurrentes, cuyos resultados preliminares indican una mejor respuesta del IR-CHOP en pacientes con subtipo $A B C$. El beneficio de la adición de esta droga en este subgrupo específico de linfomas de alto riesgo está sustentado en el hecho de que la vía en la cual interfiere, la vía del $B C R$, está relacionado más estrechamente en la patogénesis de los LDCBG ABC que en los GCB. ${ }^{(33)}$ Actualmente se vienen desarrollando ensayos clínicos aleatorizados para consolidar su beneficio en primera línea de pacientes con características de alto riesgo. El más importante es el estudio fase 3 PHOENIX ${ }^{(34)}$, que utiliza ibrutinib más R-CHOP (IR-CHOP) vs R-CHOP para pacientes nuevos con LDCBG no GCB y cuyos resultados saldrán en los próximos años.

\section{BoR-CHOP}

Un inhibidor de proteosomas como el bortezomib, fue una de las primeras drogas estudiadas. Su seguridad y eficacia en combinación con R-CHOP ha sido evaluado en diversos estudios de fase 1 como de fase 2 como esquema de primera línea en este grupo de pacientes ${ }^{(35,36)}$. Sin embargo, existen estudios randomizados con resultados preliminares ya publicados que parecen indicar que esta combinación no es superior al R-CHOP para el manejo de LDCBG de alto riesgo. El estudio multicéntrico fase 2 PYRAMID ${ }^{(37)}$ sugiere en su análisis intermedio que no hay una ventaja estadísticamente significativa al adicionar bortezomib al R-CHOP (después de un seguimiento de 31,5 meses se encontró un SLG y SG a los 2 años: $77 \%$ vs $82 \%$ y $80 \%$ vs $82 \%$ para el R-CHOP y BoRCHOP respectivamente). No obstante que los resultados señalados parecen no demostrar un beneficio concluyente está en curso el estudio fase 3 REMoDL-B ${ }^{(38)}$ que compara la eficacia del VR-CHOP vs RCHOP en pacientes con LCBGD según la clasificación molecular.

\section{EveR-CHOP}

Los inhibidores del mTOR, sobre todo el everolimus, han sido principalmente estudiadas en linfomas de células $\mathrm{B}$ refractarios o recurrentes a una primera línea ${ }^{(39-40)}$, demostrando su utilidad en este contexto. Recientemente, en un estudio fase 1 (NCCTG 1085) (Alliance) ${ }^{(41)}$ se investigó la seguridad y eficacia del everolimus cuando se adiciona al R-CHOP (EveR-CHOP) en pacientes con LDCBG nunca tratados, encontrando que la combinación es segura en primera línea y dejando la puerta abierta para el inicio de estudios fase 2 en el subgrupo de pacientes con LDCBG de alto riesgo.

\section{CONCLUSIONES}

Los linfomas no Hodgkin tienen una alta incidencia en nuestro país, con una proporción de casos nuevos que han ido incrementando con los años, convirtiéndose poco a poco en un problema de interés para la salud pública. Al enfrentar a los LDCBG nos encontramos ante una enfermedad heterogénea, con diferentes características clínicas, de agresividad, pronóstico y respuesta al tratamiento.

Es necesario contar con un sistema de clasificación que nos permita poder agruparlos en linfomas de alto y bajo riesgo y así optar por estrategias terapéuticas diferenciadas. Este agrupamiento está permitiendo el desarrollo de alternativas terapéuticas para los linfomas de alto riesgo que equiparen a los buenos resultados obtenidos en los pacientes de bajo riesgo.

El R-CHOP es el tratamiento estándar en los linfomas de bajo riesgo, sin embargo, el esquema R-DA-EPOCH, parece ser la estrategia actual más adecuada en linfomas de alto riesgo, a la espera de nuevas combinaciones como ibrutinib, lenalidomida, bortezomib y everolimus con las que se esperan resultados más alentadores.

Contribuciones de autoría: PCR, FVR, RSJ, EPC y FSS, han participado en la concepción del artículo, la recolección de datos, búsqueda de información, su redacción y aprobación de la versión final.

Fuentes de financiamiento: autofinanciado.

Conflictos de interés: los autores manifiestan no tener conflictos de interés en la publicación del artículo.

\section{REFERENCIAS BIBLIOGRÁFICAS}

1. FerlayJ,Soerjomataram I,ErvikM,Dikshit $\mathrm{R}$, Eser S, Mathers C, et al. GLOBOCAN 2012 v1.0, Cancer Incidence and Mortality Worldwide: IARC CancerBase No. 11 [Internet]. Lyon, France: International Agency for Research on Cancer; 2013. (citado el 15 de diciembre de 2016). Disponible en: http://publications.iarc.fr/ Databases/Iarc-Cancerbases/Globocan2012-Estimated-Cancer-IncidenceMortality-And-Prevalence-Worldwide-In2012-V1-0-2012
2. Lu TX, Miao Y, Wu JZ, Gong QX, Liang $\mathrm{JH}$, Wang $\mathrm{Z}$, et al. The distinct clinical features and prognosis of the $\mathrm{CD}_{10} \mathrm{MUM1}^{+}$and $\mathrm{CD} 10{ }^{-} \mathrm{Bcl}-\mathrm{MUM1}$ diffuse large B-cell lymphoma. Sci Rep. 2016;6:20465. doi: 10.1038/srep20465. 
3. Nowakowski GS, Czuczman MS $\mathrm{ABC}, \mathrm{GCB}$, and Double-Hit Diffuse Large B-Cell Lymphoma: Does Subtype Make a Difference in Therapy Selection? Am Soc Clin Oncol Educ Book. 2015:e449-57. doi: 10.14694/ EdBook_AM.2015.35.e449.

4. Datos Epidemiologicos. [Internet]. Lima: Instituto Nacional de Enfermedades Neoplasicas (INEN); 2015 [citado 20 de Diciembre de 2016]. Disponible en: http://portal.inen.sld.pe/indicadoresanuales-de-gestion-produccionhospitalaria/

5. Padilla Valdez JJ, Ulloa Pérez V, Venegas Ojeda D. Características epidemiológicas, clínicas y patológicas de los linfomas en el Hospital Nacional Cayetano Heredia del año 1998 al 2008. Acta méd peruana. 2011;28(1):12-8.

6. Alegría-Valdivia ER, Grados-Doroteo J, Rodríguez-Hurtado D. Características clínicas de los linfomas no Hodgkin en el Hospital Nacional Arzobispo Loayza. Rev Soc Peru Med Interna. 2012;25(1):26-30.

7. Swerdlow SH, Campo E, Pileri SA, Harris NL, Stein H, Siebert R, et al. The 2016 revision of the World Health Organization classification of lymphoid neoplasms. Blood. 2016;127(20):237590.doi: 10.1182/blood-2016-01-643569.

8. Carbone A, Gloghini A, Kwong YL, Younes A. Diffuse large B cell lymphoma: using pathologic and molecular biomarkers to define subgroups for novel therapy. Ann Hematol. 2014;93(8):126377. doi: 10.1007/s00277-014-2116-y.

9. Choi WW, Weisenburger DD, Greiner TC, Piris MA, Banham AH, Delabie J, et al. A New Immunostain Algorithm Classifies Diffuse Large B-Cell Lymphoma into Molecular Subtypes with High Accuracy. Clin Cancer Res. 2009;15(17):5494-502. doi: 10.1158/1078-0432.CCR-09-0113.

10. Meyer PN, Fu K, Greiner TC, Smith LM, Delabie J, Gascoyne RD, Ott G, et al. Immunohistochemical Methods for Predicting Cell of Origin and Survival in Patients With Diffuse Large B-Cell Lymphoma Treated With Rituximab. J Clin Oncol. 2011;29(2):200-7. doi: 10.1200/JCO.2010.30.0368.

11. Alizadeh AA, Eisen MB, Davis RE, Ma C, Lossos IS, Rosenwald A, et al. Distinct types of diffuse large B-cell lymphoma identified by gene expression profiling. Nature. 2000;403(6769):503-11. doi: $10.1038 / 35000501$.
12. Ziepert M, Hasenclever D, KuhntE, Glass B, Schmitz N, Pfreundschuh M, et al. Standard International Prognostic Index Remains a Valid Predictor of Outcome for Patients With Aggressive CD20+ B-Cell Lymphoma in the Rituximab Era. J Clin Oncol. 2010;28(14):2373-80. doi: 10.1200/JCO.2009.26.2493.

13. Broyde A, Boycov O, Strenov Y, Okon E, Shpilberg O, Bairey O. Role and prognostic significance of the $\mathrm{Ki}-67$ index in non-Hodgkin's lymphoma. Am J Hematol. 2009;84(6):338-43. doi: 10.1002/ajh.21406.

14. Li ZM, Huang JJ, Xia Y, Zhu YJ, Zhao W, Wei WX, et al. High Ki-67 expression in diffuse large B-cell lymphoma patients with non-germinal center subtype indicates limited survival benefit from R-CHOP therapy. Eur J Haematol. 2012;88(6):510-7. doi: 10.1111/j.16000609.2012.01778.x.

15. Aukema SM, Siebert R, Schuuring E, van Imhoff GW, Kluin-Nelemans HC, Boerma EJ, et al. Double-hit B-cell lymphomas. Blood. 2011;117(8):231931.doi: 10.1182/blood-2010-09-297879.

16. Niitsu N, Okamoto M, Miura I, Hirano M. Clinical features and prognosis of de novo diffuse large B-cell lymphoma with $t(14 ; 18)$ and 8q24/c-MYC translocations. Leukemia. 2009;23(4):777-83. doi: 10.1038/ leu.2008.344.

17. Sarkozy C, Traverse-Glehen A, Coiffier B. Double-hit and double-proteinexpression lymphomas: aggressive and refractory lymphomas. Lancet Oncol. 2015;16(15):e555-67. doi: 10.1016/ S1470-2045(15)00005-4.

18. Sathyanarayanan V, Issa AK, Ahmed MA, et al. DA-EPOCH-R for high risk diffuse large B-cell lymphoma (DLBCL): The University of Texas MD Anderson Experience. En: ASH Meeting on Lymphoma Biology, 20 de junio de 2016, Colorado Springs, CO; 2016.

19. Sathyanarayanan V, Oki Y, Issa AK, Ahmed MA, Noorani M, Fanale MA, et al. High Risk Diffuse Large B Cell Lymphoma: A Comparison of Aggressive Subtypes Treated with Dose Adjusted Chemotherapy-the University of Texas MD Anderson Experience. Blood. 2016; 128(22):106 LP-106.

20. Habermann TM, Macon WR, Maurer MJ, Ketterling RP, Kurtin
PJ, Feldman AL, et al. Treatment and Clinical Outcomes of High Grade B-Cell Lymphomas with MYC and BCL2 and/or BCL6 Rearrangements (Double Hit/Triple Hit Lymphomas). Blood. 2016;128(22):155 LP-155.

21. National Comprehensive Cancer Network. Clinical Practice Guidelines in Oncology: B-cell lymphomas. V.1.2017. Washington, PA: NCCN; 2016. (citado el 7 de Diciembre de 2016). Disponible en: https://www.nccn.org/professionals/ physician_gls/pdf/b-cell.pdf

22. Sengar M, Menon H, Dangi U, Bagal B, Khattry N, Epari S, et al. Comparison Of Treatment Outcomes with EPOCHRituximab Versus CHOP-Rituximab in Patients with De-novo Intermediate and High Risk International Prognostic Index(IPI)Diffuse Large B Cell Lymphoma(DLBCL): A Single Center Retrospective Analysis. Jain $\mathrm{H}$, editor. Blood. 2013;122(21):5115 LP-5115.

23. Huang J-J, Jiang W, Li Z-M. R-EPOCH Is Superior to R-CHOP As a First-Line Regimen in De Novo DLBCL Patients with High Ki-67 Expression. Blood. 2015;126(23):5085 LP-5085.

24. Oki Y, Noorani M, Lin P, Davis RE, Neelapu SS, Ma L, et al. Double hit lymphoma: the MD Anderson Cancer Center clinical experience. Br J Haematol. 2014;166(6):891-901. doi: 10.1111/ bjh. 12982 .

25. Howlett C, Landsburg DJ, Chong EA, Snedecor SJ, Schuster SJ, Green TM, et al. Front-Line, Dose-Escalated Immunochemotherapy Is Associated with a Significant PFS (but not OS) Advantage in 401 Patients (Pts) with Double-Hit Lymphomas (DHL): A Systematic Review and Meta-Analysis. Blood. 2014;124(21):3056 LP-3056.

26. Wilson WH, sin-Ho J, Pitcher BN, Hsi ED, Friedberg J, Cheson B, et al. Phase III Randomized Study of R-CHOP Versus DA-EPOCH-R and Molecular Analysis of Untreated Diffuse Large B-Cell Lymphoma: CALGB/Alliance 50303. Blood. 2016;128(22):469 LP-469.

27. Vitolo U, Chiappella A, Franceschetti S, Carella AM, Baldi I, Inghirami G, et al. Lenalidomide plus R-CHOP21 in elderly patients with untreated diffuse large B-cell lymphoma: results of the REAL07 openlabel, multicentre, phase 2 trial. Lancet Oncol. 2014;15(7):730-7. doi: 10.1016/ S1470-2045(14)70191-3.

28. Nowakowski GS, LaPlant B, Macon WR, Reeder CB, Foran JM, Nelson GD et al. Lenalidomide Combined With R-CHOP 
Overcomes Negative Prognostic Impact of Non-Germinal Center B-Cell Phenotype in Newly Diagnosed Diffuse Large B-Cell Lymphoma: A Phase IIStudy. J Clin Oncol. 2015 Jan 20;33(3):251-7. doi: 10.1200/JCO.2014.55.5714.

29. Chiappella A, Tucci A, Castellino A, Pavone V, Baldi I, Carella AM, et al. Lenalidomide plus cyclophosphamide, doxorubicin, vincristine, prednisone and rituximab is safe and effective in untreated, elderly patients with diffuse large B-cell lymphoma: a phase I study by the Fondazione Italiana Linfomi. Haematologica. 2013;98(11):1732-8. doi: 10.3324/haematol.2013.085134.

30. Nowakowski GS, Chiappella A, Witzig TE, Spina M, Gascoyne RD, Zhang L, et al. ROBUST: Lenalidomide-R$\mathrm{CHOP}$ versus placebo-R-CHOP in previously untreated $\mathrm{ABC}$-type diffuse large B-cell lymphoma. Future Oncol. 2016;12(13):1553-63. doi: 10.2217/fon2016-0130.

31. Younes A, Thieblemont C, Morschhauser F, Flinn I, Friedberg JW, Amorim S, et al. Combination of ibrutinib with rituximab, cyclophosphamide, doxorubicin, vincristine, and prednisone (R-CHOP) for treatment-naive patients with CD20-positive B-cell non-Hodgkin lymphoma: a non-randomised, phase $1 \mathrm{~b}$ study. Lancet Oncol. 2014;15(9):1019-26. doi: 10.1016/S1470-2045(14)70311-0.

32. Wilson WH, Gerecitano JF, Goy A, de Vos S, Kenkre VP, Barr PM, et al. The Bruton's Tyrosine Kinase (BTK) Inhibitor, Ibrutinib (PCI-32765), Has Preferential Activity in the ABC Subtype of Relapsed/ Refractory De Novo Diffuse Large B-Cell Lymphoma (DLBCL): Interim Results of a Multicenter, Open-Label, Phase 2 Study. Blood. 2015;120(21):686 LP-686.

33. Choi MY, Kipps TJ. Inhibitors of B-cell receptor signaling for patients with B-cell malignancies. Cancer J. 2012;18(5):404-10.

34. Younes A, Zinzani PL, Sehn LH, Johnson PWM, Gascoyne RD, Ahmadi T, et al. A randomized, double-blind, placebo-controlled phase 3 study of ibrutinib in combination with rituximab, cyclophosphamide, doxorubicin, vincristine, and prednisone (R-CHOP) in subjects with newly diagnosed nongerminal center B-cell subtype of diffuse large B-cell lymphoma (DLBCL). J Clin Oncol. 2014;32(15_suppl):TPS8615-TPS8615.

35. Ruan J, Martin P, Furman RR, Lee SM, Cheung K, Vose JM, et al. Bortezomib Plus CHOP-Rituximab for Previously untreated diffuse large B-cell lymphoma and Mantle Cell Lymphoma. J Clin Oncol. 2011;29(6):690-7. doi: 10.1200/ JCO.2010.31.1142.

36. Furman RR, Martin P, Ruan J, Cheung YK, Vose JM, LaCasce AS, et al. Phase 1 Trial of Bortezomib Plus R-CHOP in Previously Untreated Patients With Aggressive Non-Hodgkin Lymphoma. Cancer. 2010;116(23):5432-9. doi: 10.1002/cncr.25509.

37. Leonard JP, Kolibaba K, Reeves JA, Tulpule A, Flinn IW, Kolevska T, et al. Randomized Phase 2 Open-Label Study of R-CHOP \pm Bortezomib in Patients (Pts) with Untreated NonGerminal Center B-Cell-like (NonGCB) Subtype Diffuse Large Cell Lymphoma (DLBCL): Results from the Pyramid Trial (NCT00931918). Blood. 2015;126(23):811 LP-811.
38. Davies AJ, Caddy J, Maishman T, Barrans S, Mamot C, Care M, et al. A Prospective Randomised Trial of Targeted Therapy for Diffuse Large B-Cell Lymphoma (DLBCL) Based upon Real-Time Gene Expression Profiling: The Remodl-B Study of the UK NCRI and SAKK Lymphoma Groups (ISRCTN51837425). Blood. 2015;126(23):812 LP-812.

39. Barnes JA, Jacobsen E, Feng Y, Freedman A, Hochberg EP, LaCasce AS, et al. Everolimus in combination with rituximab induces complete responses in heavily pretreated diffuse large B-cell lymphoma. Haematologica. 2013;98(4):615-9. doi: 10.3324/haematol.2012.075184.

40. Hess G, Smith SM, Berkenblit A, Coiffier B. Temsirolimus in mantle cell lymphoma and other non-Hodgkin lymphoma subtypes. Semin Oncol. 2009;36 Suppl 3:S37-45. doi: 10.1053/j. seminoncol.2009.10.012.

41. Johnston PB, LaPlant B, McPhail E, Habermann TM, Inwards DJ, Micallef IN, et al. Everolimus combined with R-CHOP-21 for new, untreated, diffuse large B-cell lymphoma (NCCTG 1085 [Alliance]): safety and efficacy results of a phase 1 and feasibility trial. Lancet Haematol. 2016;3(7):e309-16. doi: 10.1016/S2352-3026(16)30040-0.

Correspondencia: Paulo César Castañeda Ruiz Dirección: Calle Mariscal Miller 1840, Lince.

Lima, Perú.

Correo electrónico:paulocesar_cr86@hotmail.com

\section{Nuestros artículos se encuentran indizados en:}

$$
\text { Publatued }
$$

www.ncbi.nlm.nih.gov/pubmed 